Argumentation et Analyse du Discours

\title{
La correspondance comme genre éthique
}

Correspondance as an Ethical Genre

Anna Jaubert

\section{OpenEdition}

Journals

Édition électronique

URL : http://journals.openedition.org/aad/985

DOI : 10.4000/aad.985

ISSN : 1565-8961

Éditeur

Université de Tel-Aviv

Référence électronique

Anna Jaubert, «La correspondance comme genre éthique », Argumentation et Analyse du Discours [En ligne], 5 | 2010, mis en ligne le 20 octobre 2010, consulté le 23 septembre 2019. URL : http:// journals.openedition.org/aad/985; DOI : 10.4000/aad.985

Ce document a été généré automatiquement le 23 septembre 2019.

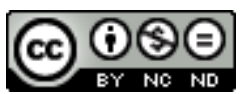

Argumentation \& analyse du discours est mis à disposition selon les termes de la licence Creative Commons Attribution - Pas d'Utilisation Commerciale - Pas de Modification 4.0 International. 


\title{
La correspondance comme genre éthique
}

\author{
Correspondance as an Ethical Genre
}

\author{
Anna Jaubert
}

1 Cet article prolonge la réflexion d'une étude précédente, «L'ethos de l'épistolier au miroir de l'autre» (Jaubert 2010: 73-88), pour faire de la composante éthique du discours le fondement du genre épistolaire. L'adjectif éthique est à entendre ici dans sa pleine polysémie, rattachable à deux substantifs, celui d'ethos, ou d'image de soi construite par le discours (Amossy 1999), et celui d'éthique comme ensemble de règles morales. Evidemment ces notions entretiennent un lien étroit; elles conditionnent fortement les correspondances d'auteurs, qui sont à la fois des correspondances réelles, constituées de lettres qui ont "voyagé " entre des êtres de chair et d'os, et des correspondances engagées dans un processus de littérarisation du fait d'au moins un des épistoliers, reconnu comme une personnalité du monde des lettres. Le rôle de l' ethos, qui projette une figure imaginaire, est une détermination générique qui marque l'épistolaire, qu'il soit un discours réel ou une transposition fictionnelle. Les caractéristiques d'un genre de discours découlent de la situation de communication dans laquelle il s'inscrit, et qu'il reflète dans son cadre énonciatif. Dans le cas des correspondances, cette situation est celle d'une interaction très particulière : d'un côté l'échange verbal est direct entre les épistoliers, mais comme il est pratiqué hors la vue et avec un temps de réponse différé, il est aussi conditionné par son statut de discours écrit, qui fait de lui un discours littérarisable. Là, les tours d'écriture sont allongés, les actes de langage complexifiés, et par-dessus tout l'ethos de l'épistolier prend une importance décisive. En me fondant sur le décalage pragmatique à l'œuvre dans ce type d'échange, je montrerai qu'il peut constituer un genre de discours définissable par son ancrage éthique, aussi bien dans le dire que dans le dit. Nous verrons qu'un fonds de valeurs partagées est au cœur de son dispositif, et comment il tend à produire des idéaux. Cette observation conduira à interroger précisément la littérarisation des lettres, liée au contexte socio-discursif de l'époque classique ${ }^{1}$, autrement dit de la 
période qui couvre les $17 \mathrm{e}$ et $18 \mathrm{e}$ siècles français, analysant le lien qui s'établit alors entre posture éthique et style des discours.

\section{Approche empirique. Les conditions d'un discours coopératif}

2 L'interprétation d'un discours ne va pas sans la prise en compte de son environnement. La pragmatique, qui a marqué le paysage des études de textes au cours des dernières décennies, se fonde sur un retour de l'intentionnalité, et conjointement, sur le rôle du contexte de la communication (Strawson 1970). Mais si une situation matérielle se reflète en effet dans le cadre énonciatif du discours (Jaubert 1990), le but de la communication, lui, n'est pas toujours explicitement avoué. Pourquoi engage-t-on une correspondance? Peut-on postuler une macro-valeur illocutoire pour ce type de discours, sachant que les lettres auxquelles nous nous intéressons ne sont pas de courts billets à visée utilitaire immédiate, mais que tout au contraire elles projettent une relation durable?

\subsection{Une demande de reconnaissance de place}

3 Vers la fin du siècle dernier François Flahault développait l'idée que la parole est toujours une " parole intermédiaire » (1978). Au-delà de son rôle informatif, elle est en charge d'une donnée fondamentale des sociétés humaines à savoir que «ses membres se confirment les uns les autres " (Watzlawick et al. 1972). L'image de soi du locuteur, son ethos, est donc la clé de la construction du discours. Il se trouve que dans une correspondance, les effets de l'interaction sont suspendus par l'absence physique de l'interlocuteur et que, de ce fait même, l'image de soi de l'épistolier peut s'élaborer à loisir, hors de toute pression de l'autre à qui il faudrait «rendre» la parole, et qui pourrait manifester son impatience ou sa mauvaise humeur devant une intervention qui s'éternise. Cette situation privilégiée découle naturellement de la temporalité spécifique de la relation épistolaire; elle se double d'une deuxième implication du temps long dans les correspondances qui s'inscrivent dans la durée, et surtout qui préméditent cette inscription ${ }^{2}$. Ici le déploiement confortable de l'ethos, et l'anticipation d'une certaine pérennité de l'échange, se conjuguent pour faire monter en première ligne la demande de reconnaissance des places. Comme dans l'interaction ces places sont complémentaires, l'image du 'Qui je suis/prétends être pour toi' dessine en creux celle d'un 'Qui tu es pour moi' (Flahault), et l'on ne dira jamais assez à quel point l'ethos projeté par l'épistolier appelle un répondant. C'est ainsi que la lettre se constitue comme lien social. Dans ces conditions, le partage des valeurs est une stratégie à la fois intuitive et très concertée de la demande de reconnaissance qui vise à garantir les conditions de félicité d'un échange ; à nos yeux, elle représente même une véritable clause de viabilité pour l'échange épistolaire. Deux cas de figure opposés ${ }^{3}$, la correspondance entre Henriette*** et Jean-Jacques Rousseau ${ }^{4}$ d'une part, celle entre Frédéric de Prusse et Voltaire ${ }^{5}$ de l'autre, permettent de s'en convaincre.

Ces deux relations ont l'avantage de présenter assez de points communs pour être confrontées. Une admiratrice et un admirateur d'une célébrité littéraire vont à sa rencontre en l'absence de tout contact antérieur, et espèrent engager avec elle un commerce durable. Mais là s'arrêtent les similitudes externes: entre Henriette et 
Rousseau, la correspondance, limitée à trois échanges ${ }^{6}$, tourne court au bout d'un an, alors que Frédéric et Voltaire, au-delà même de toute attente, s'écrivent durant $42 \mathrm{ans}^{7}$, échangeant plus de 800 lettres. Un tel contraste prend sa source dans l'accueil fondamentalement divergent fait aux demandes de reconnaissance de places, échec pour l'une, et réussite pour l'autre.

\subsection{Des valeurs comme argument}

5 La complémentarité des places postulées appelle la co-ratification des interactants. Elle implique donc l'équilibre d'une négociation où chacun doit trouver son compte. Sans doute l'inégalité sociale a-t-elle pesé dans la balance: Henriette n'avait que son admiration d'inconnue à offrir, alors que Frédéric gratifiait Voltaire d'un hommage princier. Pour autant, au vu des autres correspondants de nos auteurs, nombreux mais pas nécessairement prestigieux, cette donnée est loin de tout expliquer. En revanche le rôle des valeurs et des références partagées paraît, quant à lui, décisif. La construction de l'ethos dans son processus en témoigne clairement.

6 Celui qui fait le premier pas dessine un rapport gratifiant pour l'autre. Henriette et Frédéric motivent leur démarche par une demande de conseil ou un envoi d'ouvrages. La requête est un acte banal, mais il comporte une menace pour la face négative ${ }^{8}$ de l'allocutaire, son «territoire », que l'on n'est pas censé envahir sans bonne raison, ni offre de "compensation » symbolique : la politesse et la diplomatie en ont fait leur règle. La requête sera donc légitimée par des protestations d'estime, et surtout par l'assurance d'affinités morales qui jouent le rôle d'argument indirect en faveur des places proposées.

7 Henriette, qui veut se lancer dans des études, prépare sa demande de conseils en invoquant son admiration pour le philosophe qui « connait le cœur humain », tout en alléguant que le sien «a besoin d'une entière ouverture » qu'elle ne peut faire à n'importe qui :

je ne la [l'entière ouverture] puis faire utilement qu'à vous, Monsieur, je ne me sens cette confiance qu'en vous. Je l'ai parce que vous êtes le seul dont la manière de raisonner me plaise, me convienne, m'éclaire, me persuade; vos principes me paraissent les plus vrais et les plus solides, les plus d'accord avec la nature, l'expérience et la raison; ce que je dis, non pour vous faire un compliment, je n'ai point cette vanité, mais seulement pour justifier une témérité que le désir seul de mon bonheur a pu m'inspirer99.

8 Les valeurs convoquées sont dans l'air du temps : la connaissance du cœur et de l'esprit, dont le couple à l'époque s'était lexicalisé, l'exercice du raisonnement, la recherche du bonheur qui commence à modifier profondément les mentalités (Mauzi 1994), mais surtout une exigence de sincérité de nature à séduire le farouche défenseur d'Alceste ${ }^{10}$ : «Je ne dissimulerai pas les choses dont mon amour-propre pourrait souffrir. Je sais que je parle à un philosophe qui connaît la marche du cœur humain, et qui sait être indulgent » (ibid., 19: 242). Pourtant ces valeurs partagées, maintes fois exhibées par Henriette, n'ont pas convaincu son interlocuteur, promu confident d'élection. C'est qu'en l'occurrence la caution était elle-même sujette à caution. En fait de valeurs partagées, l'une manquait à l'appel, fragilisant l'ensemble de l'édifice éthique construit par le discours de l'épistolière. Henriette, qui connaissait la piètre opinion de Rousseau sur les «femmes savantes", croyait pouvoir plaider l'exception, mais pour ce dernier (et à son grand dam), sa requête ressemblait à une remise en cause : 
D'ailleurs, comme c'est un endroit du livre de votre Emile, qui en détruisant les idées de bonheur que je m'étais faite [sic], occasionne l'incertitude où je suis, ce m'est une raison de plus de désirer vos conseils. Qui peut mieux que vous même étendre ou restreindre vos pensées, pour en faire selon les différentes circonstances une juste application? (Ibid.).

Par le jeu des présupposés, demander à Rousseau un avis sur le genre d'étude à poursuivre, c'était l'inviter à reconnaître implicitement le bien-fondé du projet, à ouvrir pour elle une brèche dans son système de valeurs misogyne. Ce qu'elle appelle " une juste application ", revient à admettre qu'une femme libre de devoirs familiaux puisse jouer un rôle intellectuel dans la société, comme d'autres tenant des salons réputés ${ }^{11}$, l'ont fait à cette époque. Rousseau n'était pas prêt à ce genre de concession. Irrité, il dénonce la manipulation illocutoire, qu'il croit doublée d'une imposture, s'imaginant que le nom d'Henriette cachait celui de la jeune Suzanne Curchod, connue dans le milieu genevois :

Je ne prends pas le change, Henriette, sur l'objet de votre lettre, non plus que sur votre date de Paris. Vous recherchez moins mon avis sur le parti que vous avez à prendre que mon approbation pour celui que vous avez déjà pris (L. 3256, 20 : 18).

Revenu de son erreur sur la personne, Rousseau n'en modifia pas son jugement pour autant. La demande d'Henriettel'obligeait trop à reconsidérer sa conception de la société et ses idées arrêtées sur la place des femmes. La démarche malheureuse d'Henriette révèle en fin de compte une incompatibilité des valeurs qui, d'emblée, condamnait l'échange.

11 Pour Frédéric et de Voltaire, il en allait tout autrement. En proclamant son amour des lettres et son soutien envers les philosophes, le prince héritier se démarque de son père et affiche son appartenance au camp militant de son interlocuteur : comme lui, il porte haut les valeurs des Lumières. Son ethos est celui d'un admirateur, mais aussi d'un lecteur cultivé qui sait penser par lui-même. Au-delà même du propos énoncé, il montre dans son dire qu'il maîtrise le code des honnêtes gens, de la société policée «à la française $»^{12}$, maniant comme il sied le « on » qui gaze avec élégance la convocation des personnes: "Les beautés en [de vos ouvrages] paraissent nouvelles chaque fois qu'on les relit. [...] Votre Henriade me charme, et triomphe heureusement de la critique peu judicieuse que l'on a faite d'elle " (4:28-29). Ici la connivence morale et idéologique semble déjà acquise. Le combat des philosophes contre l'obscurantisme aligne ses topoï, l'hommage à celui qui "œuvre pour le bien du public » est appuyé, les valeurs de référence, comme les lieux communs qui rapprochent, se concentrent: "Tel est le destin des grands hommes: leur génie supérieur les expose toujours en butte aux traits envenimés de la calomnie et de l'envie» $(4: 28)$. Allusion flatteuse, s'il en est. Aujourd'hui de tels éloges passeraient difficilement, mais au 18e siècle ils s'inscrivent dans une politesse ordinaire, accordée à des valeurs qui privilégient fortement la distinction. Cela nous renvoie à une constante morale élitiste, promue par la vie de salon depuis ses origines: cette constante n'est autre qu'une sociabilité exigeante, entre des âmes au-dessus du commun, et qui se reconnaissent entre elles.

L'ethos est l'argument majeur et surplombant de la demande de reconnaissance de place : il s'appuie sur des valeurs partagées, si bien qu'a contrario, une discordance des valeurs peut s'avérer fatale à la poursuite de l'échange épistolaire. Mais ira-t-on au-delà du simple partage des valeurs empruntées à un interdiscours consensuel? Dans le cadre d'un échange assez harmonieux pour se prolonger, la correspondance pourrait- 
elle devenir un creuset de valeurs nouvelles, autrement dit un "laboratoire de valeurs »?

\section{La fabrique des idéaux et l'éthique du style}

13 En faisant miroiter des valeurs partagées, les correspondances intègrent évidemment les codes, et les idéaux socio-culturels de leur temps. Sans doute aussi contribuentelles, sous certaines plumes, à requalifier ces valeurs et à les déployer ${ }^{13}$. Dans le circuit qu'il informe, le discours épistolaire ferait-il davantage que de confirmer ces valeurs ambiantes, et en vient-il à produire lui-même des valeurs? Indéniablement, l'ethos convaincant qui se dessine suscite une idéalisation des sujets, et ce que l'on constate est déjà en soi une surenchère des valeurs.

\subsection{Un commerce fondé sur la « distinction »}

14 L'ethos de l'épistolier est lui-même partie prenante d'un ethos épistolaire plus ou moins prédisposé par le genre du discours. L'échange de lettres prolongé, il faut insister sur ce point, dépasse les enjeux utilitaires et devient une relation autonomisée, décalée par rapport aux affaires de la vie : pour tout dire, une relation imaginaire. Les idéaux de ceux qui tiennent la plume, pris dans l'engrenage de la confirmation réciproque, en viennent à composer des figures idéales, celles d'interactants d'un commerce privilégié entre beaux esprits, ou belles âmes.

A l'époque qui nous occupe, les correspondances sont ainsi fondées sur l'idée d'une distinction. Diderot dans ce 18e siècle de la sensibilité montante fait converger éthique et esthétique ; ses lettres à Sophie Volland sont explicitement destinées à une «tendre et solide amie ", l'amour qui s'y décline n'est jamais loin de la réflexion morale, et la réflexion morale y renvoie sans cesse à la subjectivité des affects :

Il ne faut pas qu'il y ait beaucoup de statues dans un jardin, et celui-ci m'en paraît un peu trop peuplé. Il faut regarder les statues comme des êtres qui aiment la solitude et qui la cherchent, des poètes, des philosophes et des amants, et ces êtres ne sont pas communs. Quelques belles statues cachées dans les lieux les plus écartés, les unes loin des autres, qui m'appellent, que j'aille chercher ou que je rencontre ; qui m'arrêtent et avec lesquelles je m'entretienne longtemps [...].

Nous dînâmes tous d'appétit. Notre baron, le nôtre, fut d'une folie sans égale. Il a de l'originalité dans le ton et dans les idées. Imaginez un satyre gai, piquant, indécent et nerveux, au milieu d'un groupe de figures chastes, molles et délicates. Tel il était entre nous. Il n'aurait ni embarrassé ni offensé ma Sophie, parce que ma Sophie est homme et femme, quand il lui plait. Il n'aurait ni offensé ni embarrassé mon ami Grimm, parce qu'il permet à l'imagination ses écarts, et que le mot ne lui déplaît que quand il est mal placé. o combien il fut regretté, cet ami! que ce fut un intervalle dans notre repas bien doux que celui où nos âmes s'ouvrirent [...] (Lettre du 10 mai 1759, Diderot $1956:$ 135-37).

16 Ce que l'on observe dans ces pages, et qui se confirme dans l'ensemble des Lettres à Sophie Volland, c'est l'imbrication étroite entre l'épanchement, les états d'âme de l'amant, et l'arrière-plan du microcosme des familiers. On sait qu'à l'époque, comme au siècle précédent, les lettres circulent, sont lues publiquement dans les salons et les cercles d'amis. Par anticipation, le rapport interindividuel dérive en rapport social. Les images de soi construites intègrent le regard d'un destinataire additionnel ${ }^{14}$, la petite société choisie, garante des valeurs qui deviennent les insignes de l'appartenance à un 
groupe d'êtres, « des poètes, des philosophes, et des amants[qui] ne sont pas communs ", qui se comprennent et qui s'apprécient.

Il s'agit dès lors avant tout de parler le même langage. De fait, le rendez-vous avec l'Autre a bien lieu dans le langage. Il est aussi un rendez-vous avec le langage (Jaubert 1987 : 344 et suiv.). Dans l'interaction, les stéréotypes ${ }^{15}$ acquièrent une fonction préargumentative: ils produisent des énoncés reconnaissables au sens plein du terme, c'est-à-dire assurés d'être reconnus, puisqu'ils sont l'expression de valeurs communes déjà stabilisées par leur mise en circulation. Alors, le choix opéré dans la palette des stéréotypes disponibles devient significatif des valeurs auxquelles l'épistolier entend adosser son discours : on l'a vu avec Henriette comme avec Frédéric. La sélection des stéréotypes caractérise si bien le discours épistolaire que la scénographie du "genre second » correspondant (Bakhtine 1984 : 267), en l'occurrence le roman épistolaire, sa transposition littéraire et fictionnelle, tend à récupérer ce trait basique de la connivence.

Ainsi la Nouvelle Héloïse de Rousseau a-t-elle su toucher un large public gagné par l'utopie d'une harmonie préétablie dans le langage. Le plus grand succès de librairie de son temps, se présente comme une «longue romance » où le discours coule de source, intarissable, et avec un faible contrôle du métadiscours, car « c'est ainsi que le cœur sait parler au cœur » (Rousseau $1961: 15$ ).

19 A l'opposé, et avec la systématicité du pastiche, Les liaisons dangereuses manient l'hyperréflexivité langagière comme un signe de l'innocence perdue. La marquise de Merteuil et le Vicomte de Valmont construisent leur ethos cynique en faisant valoir une conscience linguistique affûtée, où les formules fétiches des roués affichent leur quête du divertissement, du " plaisant », et surtout un cinglant mépris des autres :

Peut-être, si vous eussiez connu cette femme plus tôt, en eussiez-vous pu faire quelque chose; mais cela a vingt deux ans, et il $\mathrm{y}$ en a près de deux qu'elle est mariée. Croyez-moi, Vicomte, quand une femme s'est encroûtée à ce point, il faut l'abandonner à son sort ; ce ne sera jamais qu'une espèce (Laclos $1951: 19$ ).

Le pronom neutre "cela ", qui dans la première lettre de la Marquise désignait déjà Cécile, s'associe au délocutif «espèce». Ensemble ils stigmatisent dans l'esprit des libertins l'inconsistance de leurs victimes. Quant à l'emploi de l'italique (dans le texte ici), il souligne l'usage concerté d'un mot à la mode qui, pour souder la coterie des uns, prononce l'exclusion des autres.

21 Ainsi les libertins connaissent-ils le prix de la maîtrise des codes. C'est le catéchisme de la débauche enseigné par Valmont à Cécile qui, d'emblée, saura convaincre Gercourt de son infortune :

J'occupe mon loisir [...] à composer une espèce de catéchisme de débauche à l'usage de mon écolière. Je m'amuse à n'y rien nommer que par le mot technique ; et je ris d'avance de l'intéressante conversation que cela doit fournir entre elle et Gercourt la première nuit de leur mariage (ibid., 256-257).

22 Et pour Madame de Merteuil le style se mesure à l'aune des artifices déployés :

Un mot encore. Voyez donc à soigner votre style. Vous écrivez toujours comme un enfant [...] c'est que vous dites tout ce que vous pensez, et rien de ce que vous ne pensez pas. Cela peut passer ainsi de vous à moi [...] mais avec tout le monde ! avec votre amant surtout ! (Ibid., p. 242).

23 On change résolument de ton avec la correspondance passionnée que Julie de Lespinasse entame avec Guibert, parti courir l'Europe, et qui se veut, comme souvent, 
un défi à l'absence. La langue pratiquée est naturellement celle de l'intimité, d'une intimité devenue la valeur et la fin en soi du discours qui prolonge et qui retend les liens de la conversation. Cette langue puise largement dans l'intertexte de la longue causerie qu'elle entretient, intégrant du discours rapporté indirect :

Vous me dites que vous êtes agité de regrets, de remords même; que votre sensibilité n'est que douleur ; [...] il me semble que vous parlez de ce que vous avez senti, non de ce que vous sentez.

Les gens qui sont gouvernés par le besoin d'aimer [...] ne disent point qu'ils rentreront dans leur âme pour y trouver ce qu'ils aiment ; ils croient ne l'avoir pas quitté (Lespinasse s. d. : 8, 10).

- ou mieux encore, des reformulations directes : «Mes lettres vous sont nécessaires, cela peut-il être vrai ? oui, puisque vous le dites " (ibid., 25). Ici, la recherche perpétuelle d'une adéquation des mots à une sensibilité exacerbée (Siess 1998), traduit la quête d'un supplément d'âme pour une relation amoureuse qui conserve la blessure de l'inassouvissement, mais qui en même temps se console dans la magie phatique du discours adressé.

On comprend dès lors que le commerce épistolaire, grisant, crée une espèce de dépendance que les amants sont parfois tentés de prolonger, même lorsque la séparation physique a pris fin. Ce fut le cas pour Julie de Lespinasse. On comprend aussi qu'en sacrifiant au culte de la transparence des âmes, certaines correspondances passionnées aient donné une expression possible, et à travers elle un accomplissement, à une relation fusionnelle dont on ignore finalement si elle est révélée ou créée par la lettre, en tout cas où le dire aspire au faire être durable, et parfois y parvient. La relation entre le Chevalier de Boufflers et Madame de Sabran en est un exemple célèbre.

Manifestement ces correspondances cristallisent des valeurs préexistantes, elles offrent une caisse de résonance aux groupes qui les ont secrétées, mais on ne les voit pas (et on les imagine mal) directement produire des valeurs inédites, court-circuitant l'étape de la décantation sociale ${ }^{16}$. En revanche, leur engagement dans la pérennité de l'écrit leur promet une valeur ajoutée, en tant que productions verbales. «Le scripturaire a vocation au littéraire» rappelle G. Molinié (2008): l'enjeu éthique de l'intercompréhension se double d'un enjeu esthétique. L'attention au bien dire, dont Laclos révèle la face pervertie en même temps que la puissante performativité, détermine en profondeur le discours de la lettre : à un certain moment culturel, il a sans nul doute contribué à élargir l'assiette de la littérarité.

\subsection{La motivation éthique du style}

En sortant de la sphère technique des secrétaires attitrés au service des grands, l'art de la correspondance a gagné la sphère mondaine où il est devenu l'affaire des «beaux esprits $»^{17}$.

La composante éthique du discours épistolaire se déclare en fait à deux niveaux, celui $\mathrm{du}$ dit et celui du dire. La dominante affective et morale, accordée à sa situation de discours privé, ne présume pas d'une forme qui serait sentencieuse. Certes, les lettres sentencieuses existent, mais par nature le genre épistolaire est des plus ramifiés ${ }^{18}$. L'aveu de faiblesse et la demande d'excuse, rituels des épistoliers qui plaident coupables en reconnaissant leur fâcheux penchant à moraliser, disent au contraire que 
là n'est pas le ton le plus goûté : Julie de Wolmar assume l'épithète de "prêcheuse " au terme d'une longue épître ("Ici finissent les sermons de la prêcheuse » (IIIe partie, Lettre 20), et par ailleurs Rousseau projette sur la lettre un idéal de légèreté qui lui fait défaut :

Je réussis mieux aux ouvrages qui demandent du travail, qu'à ceux qui veulent être faits avec une certaine légèreté, comme les lettres; genre dont je n'ai jamais pu prendre le ton, et dont l'occupation me met au supplice (Rousseau 1959 : 114).

La question qui se pose est celle de la posture appropriée du scripteur. De fait, le discours que Rousseau n'arrive pas à tenir est celui de l'indispensable légèreté de l'être dans son rapport à l'autre, celui d'une délicatesse exigeante héritée de la galanterie du 17e siècle et qui s'est illustrée sous des plumes brillantes : on pense à Voiture, à Guez de Balzac, à Madame de Sévigné. Un style enjoué se fait la marque de la politesse du cœur. Voiture, orfèvre en la matière, tourne en dérision la topique du discours amoureux (la séparation cause de mort, la cruauté de l'aimée, etc.), dans une déclaration badine, libérée de son habituel enjeu : feignant d'écrire post mortem un faire-part de son propre décès, il annonce à Julie d'Angennes qu'il est mort à l'instant même de son départ, mais, ajoute-t-il plaisamment, à l'insu de tout le monde, et sans avoir voulu être trop vite enterré, car " [il a] toujours eu aversion à cela $»^{19}$ ! La pochade est galante, qui gratifie la destinataire en lui épargnant la pression attachée aux déclarations d'amour. A la cour comme à la ville, l'honnêteté est une valeur éminemment conversationnelle. Il s'agit de savoir parler à l'autre un langage qu'il peut entendre ${ }^{20}$, et qu'il lui est agréable d'entendre. Le savoir correspondre est un savoir plaire, et le savoir plaire est un savoirvivre.

La construction d'un ethos si parfaitement accordé au destinataire, et si fidèle à nos rapports humains différents, tire profit du décalage de l'écrit. Les lettres de La Haye, réellement écrites par Diderot à son retour de Russie en avril 1774, sont un bel exemple des variations motivées par un destinataire particulier ${ }^{21}$. Dans la gamme de leur choix thématiques et modalisateurs, ces lettres révèlent clairement l'opération officieuse, plus dialogique que dialogale (Jaubert, $2005: 215-230$ ), à laquelle nous nous livrons lorsque nous écrivons une lettre : une opération qui chaque fois vise à configurer, ou à reconfigurer, les images complémentaires des interlocuteurs.

31 Par un effet rétroactif, simuler la communication fait sens. Si Diderot transpose dans ses autres écrits, notamment dans ses œuvres philosophiques, le style interactif de la lettre, c'est qu'il ressent la nécessité d'ancrer son argumentation dans une relation humaine sensible, de se donner un interlocuteur à émouvoir pour mieux le convaincre. Bref, à inscrire dans son discours la figure d'un actant concerné.

L'éthique du genre peut alors être pensée comme l'assomption de son ambivalence. Discours en prise sur la "vraie vie», il est aussi dans un décalage qui lui en fait transcender les visées immédiates, lui permet de s'inscrire dans la durée, dans l'exemplarité, et par surcroît d'offrir l'image d'un talent. Il n'est pas surprenant que la lettre ait été perçue comme un marchepied pour l'entrée dans la République des (Belles)-Lettres (Jaubert 2006 : 137-148). Henriette s'appliquait à bien écrire pour être reconnue par Rousseau, mais elle avait aussi recopié leur correspondance pour la publier. Voltaire et Frédéric corrigeaient certaines de leurs lettres en vue de leur impression, et Voltaire voyait en elles « de bien singuliers mémoires pour servir un jour à l'histoire du siècle » (D. 2573, lettre du 22 déc. 1741, $8: 150$ ). Car c'est à la réception que le genre, situé entre deux champs, est littérarisable ${ }^{22}$. Mais il l'est d'autant plus 
sûrement qu'ici les deux champs, le mondain et le littéraire, sont en large intersection, et que la littérarisation de la lettre est anticipée. Les épistoliers, conscients de s'adresser d'une part à des connaisseurs qui apprécieront leur talent et d'autre part peut-être à la postérité, ont soigné leur écriture, y compris en la parant des charmes du naturel.

De ces observations je conclurai qu'à l'éthique de la correspondance engagée s'attache un certain style. Plus que laboratoire de valeurs, la lettre apparait d'abord et essentiellement comme le laboratoire d'une valeur : celle de la congruence des façons de dire avec la posture que l'épistolier adopte, comme être humain et comme être social. Venant à inspirer le sentiment d'un certain bonheur de la parole, cette congruence peut promouvoir la lettre au rang de petite œuvre. Alors la littérarité indécise d'un genre intermédiaire a pour un temps donné une forme reconnue à la paratopie d'un écrivant-écrivain ${ }^{23}$.

\section{BIBLIOGRAPHIE}

Adam, Jean-Michel. 1998. " Les genres du discours épistolaire », Siess, Jürgen (éd.). La Lettre entre réel et fiction (Paris : Sedes), 37-53

Amossy, Ruth \& Anne Herschberg-Pierrot. 1997. Stéréotypes et clichés. Langue, discours, société (Paris : Nathan)

Amossy, Ruth. 1999. Images de soi dans le discours. La construction de l'ethos (Lausanne : Delachaux \& Niestlé)

Bakhtine, Mikhail. 1984. Esthétique de la création verbale (Paris : Gallimard)

Bossis, Mireille (éd.). 1990. L'épistolarité à travers les siècles. Geste de communication et/ou d'écriture. Actes du Colloque de Cerisy, 1987 (Stuttgart : Steiner)

Brown, Penelope \& Steven Levinson 1987. Politeness. Some universals in language use (Cambridge : CUP)

Bury, Emmanuel. 1996. Littérature et politesse. L'invention de l'honnête homme (1580-1750) (Paris : PUF)

CAIEF 1987, 39 « L'Art épistolaire »

Choderlos de Laclos. 1951. CEuvres complètes (éd. Allem) (Paris : Gallimard)

Craveri, Benedetta. 2002. L'âge de la conversation (Paris : Gallimard)

Diderot, Denis. 1956. Correspondance, éd. Georges Roth (Paris : Minuit), vol. 2

Faret, Nicolas. 1925 [1630]. L'Honneste homme ou l'Art de plaire à la cour , éd. Maurice Magendie

(Paris : PUF)

Flahault, François. 1978. La parole intermédaire (Paris : Seuil)

Goffman, Erving. 1974. Les rites d'interaction (Paris : Minuit)

Goffman, Erving. 1987, «L'ordre de l'interaction », Sociétés 14, 8-16 
Haroche-Bouzinac, Geneviève. (éd.) 1999. Lettre et réflexion morale. La lettre, miroir de l'âme (Paris : Klincksieck)

Jaubert Anna. 1987. Étude stylistique de la correspondance entre Henriette ${ }^{* * *}$ et J.-J. Rousseau. La subjectivité dans le discours (Genève \& Paris : Slatkine-Champion)

Jaubert, Anna. 1990. La lecture pragmatique (Paris : Hachette)

Jaubert, Anna. 2005. « Dialogisme et interaction épistolaire », Bres, Jacques, Pierre-P. Haillet, Sylvie Mellet, Henning Nølke \& Laurence Rosier (éds). Dialogisme et polyphonie : approches linguistiques (Paris : Duculot), 215-230

Jaubert, Anna. 2006. « De l'écriture de soi à la littérarisation, l'enjeu du style », Diaz, Brigitte \& Jürgen Siess (éds). L'épistolaire au féminin (Caen : P. U. de Caen), 137-148

Jaubert, Anna. 2010. «L'ethos de l'épistolier au miroir de l'autre » (Paris : L'Harmattan), 73-88

Lespinasse, Julie de (s.d.). Lettres (Paris : Garnier)

Lignereux, Cécile. Une écriture de la tendresse au XVIIe siècle. Pour une étude stylistique des Lettres de Madame de Sévigné (Sorbonne, 26 novembre 2009)

Maingueneau, Dominique. 1993. Le contexte de l'œuvre littéraire (Paris : Dunod)

Mauzi, Robert. 1994 [1960]. L'idée du bonheur dans la littérature et la pensée françaises au XVIII siècle (Paris : Albin Michel [Colin])

Méré, Chevalier de. 1930. Cuvres complètes (Paris : Roches), vol. 1

Mervaud, Christine. 1985, Voltaire et Frédéric II : une dramaturgie des lumières. 1736-1778 (Oxford : Voltaire Foundation)

Molinié, Georges. 2008. «Linguistique du texte et de l'écrit. Stylistique », CMFL08, archive ouverte HAL

Mornet, Daniel. 1940. Histoire de la littérature française classique (Paris : Colin)

Rollin, Sophie. 2006. Le style de Vincent Voiture. Une esthétique galante (Saint-Etienne : Publications de l'U. de Saint-Etienne)

Rousseau, Jean-Jacques. 1973-1981. Correspondance complète, éd. R.A. Leigh (Oxford : Voltaire Foundation)

Rousseau, Jean-Jacques. 1959-1961. OEuvres complètes 1-2 (éd. Gagnebin \& Raynaud) (Paris : Gallimard)

Seguin, Jean-Pierre. 1978. «L'illusion du destinataire chez Diderot : un aspect de la stratégie persuasive », Stratégies discursives, Lyon, PUL, 217-233

Siess, Jürgen, 1998, «Effusion amoureuse et échange intellectuel. La pratique épistolaire de Julie de Lespinasse », Planté, Christine (éd.). L'épistolaire un genre féminin? (Paris : Champion)

Strawson, Peter F. 1970. « Phrase et acte de parole », Langages 17, 19-33

Voltaire. 1969-1971. Correspondance complète, éd. Theodor Besterman (Genève : Institut et Musée Voltaire), 1971-1977 (Oxford : The Voltaire Foundation)

Watzlawick, Paul, Janet Helmick Beavin \& Don D. Jackson.1972-1979. Une logique de la communication (Paris : Seuil) 


\section{NOTES}

1. La thèse de $\mathrm{C}$. Lignereux, Une écriture de la tendresse au XVIIe siècle. Pour une étude stylistique des Lettres de Madame de Sévigné (Sorbonne 2009), montre que certains textes ont été attirés a posteriori, et abusivement, dans l'orbite classique.

2. On pressent bien qu'il en va tout autrement du courrier électronique, dont la situation de communication est hybride : en conjuguant un support écrit et la possibilité d'interagir en temps réel, il crée un nouveau genre d'écrit, archivable ou jetable, mais dans les deux cas peu disposé à la littérarisation.

3. La confrontation de ces deux cas a été détaillée par A. Jaubert (2010).

4. Correspondance (éd. Leigh), t. 19 à 24 et t. 38.

5. Correspondance (éd. Besterman) 1969-1971 et 1971-1977.

6. Pour être précis, trois lettres d'Henriette reçurent une réponse, la 4e n'en eut pas, et six ans plus tard Rousseau réagit à une nouvelle démarche d'Henriette par une cinglante lettre de rupture.

7. De 1736 à la mort de Voltaire en 1778

8. Voir les FTAs (Face Threatening Acts) de Brown et Levinson 1987.

9. Lettre 3192 (19: 240). Ici, comme par la suite, les italiques à l'intérieur d'une citation correspondent à mon soulignement.

10. On se souvient de la radicalité de Rousseau sur la question, dans sa Lettre à d'Alembert sur les spectacles.

11. On se reportera utilement à Benedetta Craveri (2002) pour des portraits et pour une réflexion sur le rôle social de ces prestigieuses salonnières.

12. Au 18e siècle elle a représenté un idéal pour l'aristocratie allemande : Paris était une étape obligée lors du Grand Tour qui couronnait l'éducation des jeunes nobles.

13. Le recueil dirigé par G. Haroche-Bouzinac montre bien la place et les diverses modalités du discours moral dans des correspondances célèbres, censément privées.

14. La mise en scène du discours a été souvent relevée dans les correspondances littéraires, qui, à l'instar du langage dramatique, pratiquent la «double adresse». C. Mervaud y voit la détermination majeure de la correspondance entre Frédéric et Voltaire, qualifiée de «dramaturgie des Lumières".

15. Concernant le fonctionnement et le rôle social des stéréotypes, voir Amossy \& Herschberg Pierrot, (1997).

16. C. Lignereux (thèse citée) montre bien que la valeur d'intimité projetée par le style communicationnel inédit des Lettres de Mme de Sévigné à sa fille, se construit sur les codes et les idéaux socio-culturels de son temps.

17. D. Mornet $1940: 318$. Voir aussi E. Bury (1996).

18. Il convient même de souligner la pluralité des genres épistolaires - cf. J.-M. Adam (Siess $1998: 37-53)$.

19. Lettre $\mathrm{n}^{\circ} 123$ à mademoiselle de Rambouillet, Voiture, CEuvres t. I. p. 336-339, citée par S. Rollin (2006 : 324-329).

20. Recommandation que l'on trouve dans tous les manuels de civilité et d'art de la conversation chez Nicolas Farest comme auprès du Chevalier de Méré.

21. Ces lettres, adressées à sept correspondants différents (le Dr. Clerc, l'impératrice Catherine II, les dames Volland, M. $\mathrm{M}^{* * *}$, Mme Diderot, Mme d'Epinay, M. et Mme Vandeul) ont été analysées par J.-P. Seguin (1978: 217-233).

22. Il l'est grâce à travers la perception majorée de leur point commun, le décalage pragmatique, «trope fondateur de la littérarité » issu d'une interaction distendue (Jaubert 1990 : 212-215). 
23. On sait comment D. Maingueneau a développé le concept de paratopie de l'écrivain, « qui nourrit son œuvre du caractère radicalement problématique de son appartenance au champ littéraire et à la société » (1993: 27).

\section{RÉSUMÉS}

Les caractéristiques d'un genre de discours découlent de la situation de communication dans laquelle il s'inscrit, et qu'il reflète. Pour une correspondance, et singulièrement pour une correspondance d'auteurs, cette situation est celle d'une interaction à la fois directe et biaisée qui engage l'ethos de l'épistolier. Nous analysons les idéaux qu'il produit sur un fond de valeurs partagées. Le champ d'investigation retenu est celui de certaines correspondances célèbres du 18 e siècle, car la lettre est à cette époque une manifestation très accomplie de la sociabilité. De ce fait, elle a connu une dérive littérarisante, source d'un décalage pragmatique qui l'attire dans la mouvance d'un genre second, la correspondance littéraire, où l'implication éthique du discours devient sa détermination majeure.

The characteristics of a genre of discourse depend upon its specific situation of communication the genre being embedded in this situation and, at the same time, reflecting it. Concerning correspondences, especially between authors, this situation consists of an interaction both direct and indirect, involving the ethos of the letter writer. Our analysis focuses on the ideals it produces on a background of shared values. The field of inquiry is a choice of famous correspondences of the 18th century, since the letter was at that time an accomplished manifestation of sociability. As such, it adopted a literary form, which created a pragmatic gap and put it under the dependence of another genre, the fictional correspondence, in which ethic implications became its determining factor.

\section{INDEX}

Mots-clés : ethos, genre de discours, pragmatique, sociabilité, valeur

Keywords : ethos, genre of discourse, pragmatic, sociability, value

\section{AUTEUR}

\section{ANNA JAUBERT}

Université de Nice-Sophia Antipolis, BCL/MSH 